\title{
Evaluating the Maturity of CPS in Discrete Manufacturing Shop-Floor: A Group AHP Method with Fuzzy Grade Approach
}

\author{
Qingmeng TAN*, Yifei TONG**, Shaofeng WU***, Dongbo LI**** \\ *School of Mechanical Engineering, Nanjing University of Science \& Technology, Nanjing 210094, China, \\ E-mail:465819860@qq.com \\ **School of Mechanical Engineering, Nanjing University of Science \& Technology, Nanjing 210094, China, \\ E-mail: tyf51129@aliyun.com \\ ***School of Mechanical Engineering, Nanjing University of Science and Technology, Nanjing 210094, China, \\ E-mail: 302764848@qq.com \\ ****School of Mechanical Engineering, Nanjing University of Science and Technology, Nanjing 210094, China, \\ E-mail:db_tyf@aliyun.com
}

crossref http://dx.doi.org/10.5755/j01.mech.24.1.18370

\section{Introduction}

Along with Industry 4.0, Smart Manufacturing has been expected to be an inspiring and feasible manufacturing paradigm to improve productivity, quality and utilization of resources, reduce operation costs and development cycle as well [1].

Shop floor is the execution level of product manufacturing which also underlines the fundamental and crucial facet concerning SM implementation. The concept of Smart shop-floor can be defined as a product manufacturing utility with self-control, self-scheduling and self-organization capabilities, based on real-time data acquiring, transformation and processing. Previous researches had pointed out that Cyber-Physical Systems (CPS) which fusion cyber world and physical world is the key technology to develop smart shop floors [2-4].

National Institute of Standards and Technology (NIST) defines CPS as smart system that includes interacting networks of physical and computational components [5]. Also, CPS can be defined as systems of collaborating computational entities which are in intensive connection with the surrounding physical world and its on-going processes, providing special services available on the internet [6]. Despite of different definitions regarding different application fields such as transportation, manufacturing, etc., the characteristics of CPS can be summarized as: a) the connection between cyber world and physical world; b) data exchange in real-time; c) information flows are bidirectional with close loops.

CPS in manufacturing domain has drawn many attentions recently. Cyber Physical Production Systems (CPPS) [6] or Manufacturing cyber-physical system (MCPS) [7] which converge Cyber System (CS), Information and Communication Technology (ICT) and manufacturing decompose the traditional hierarchy automation pyramid. Advanced technologies in M-CPS include Complex Event Processing, Cloud computing and virtualization, Internet of things adoption, Big data analytics \& Cyber security [7], etc. A 5C level architecture for Industry 4.0 based manufacturing systems is proposed [8], namely Connection, Conversion, Cyber, Cognition, Configuration. Meanwhile, interaction between human and machine is important concerning CPS implementation [9]. A reference model of an anthropocentric CPS which regards human as critical components was proposed and demonstrated in an assembly station [10]. However, due to complexities and heterogeneities in real manufacturing scenarios, the degree of CPS implementation in enterprises has been fairly low [11]. Until recently, few researches have addressed evaluation problem on CPS in manufacturing domain, especially in shop floor level.

This paper proposes an evolution process of CPS in discrete manufacturing which can be characterized by two-dimensional perspectives concerning MT (manufacturing technology) and ICT (information and communication technology) elements. Then, a maturity model (CPSMM) is presented to conceptualize the maturity of CPS implementation process in discrete manufacturing shop floor. The importance weights of indicators in the maturity model are acquired based on AHP with group decision method. Linguistic variables which further transform to Triangle Fuzzy Numbers (TFNs) are proposed to represent the value of indicators guided by semantic constraints. Procedures and algorithms of proposed methods are given and applied in a mechanical shop floor for illustration.

\section{Introduction}

2.1. The evolution process of CPS implementation in shop floor level

Shop floor manufacturing can be considered as a dynamic complex system with machinery, equipment, human interacting in real time. Introducing CPS in shop floor increases the degree of complexity as information, communication and control systems interoperate with physical entities at the same time [12]. Ideally, the main features of CPS in shop floor level could be characterized as: 1 . the connection and communication between machineries and equipment;2. the coordination and collaboration with regard to production and logistic processes; 3 . the continuous and interpretable information flow from orders generation to manufacturing process;4. Real-time shop floor data acquiring, fusing and processing; 5. human-machine cooperation, smart identification and forecasting, autonomous decisionmaking, etc.

Take mechanical manufacturing shop floors into account, the material and information topological structures of these kinds of discrete environments could be considera- 
bly complicated which requires highly integration of information management and control system as well as dependable timely data acquiring and communication abilities. Therefore, different from continuous systems, the development strategy and introducing methodology of CPS implementation in such discrete system required to be addressed on account of complicated interaction among cyber and physical entities.

Based on the requirements and features of CPS, this research proposes a general evolution process with regard to CPS implementation in discrete shop floors, as depicted in Fig.1, the CPS implementation can be described as an evolution process from junior to senior phase, ultimately, to the ideal smart manufacturing system in theory. The maturity of CPS in this process is characterized across two-dimensions: cyber dimension and physical dimension, which are represented by the degree of ICT and MT in Fig.1. The arrow indicates the ascending tendency of CPS maturity.

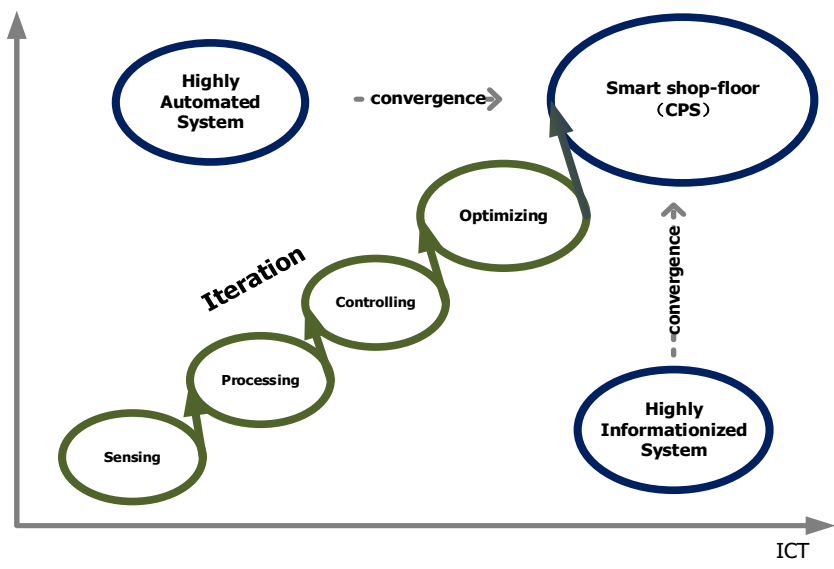

Fig. 1 Evolution process of CPS implementation in shop floor

As depicted in Fig.1, two kinds of CPS evolution paths in shop-floors are considered in this research. The first one is the iteration path which could be interpreted as a natural evolution process from sensing, processing and controlling to optimizing, ultimately to smart shop-floor with ideal CPS implementation. In this scenario, the maturity of CPS in shop-floor is iteratively ascending among different phases which measured by the degree of ICT as well as MT advancement.

The other path showed in Fig. 1 is one-dimension convergence path. Under this circumstance, the targeted shop-floor are already either highly automated systems or highly informative system. Consequently, to reach the ideal CPS phase, the feasible path would be improving the relatively insufficient aspect substantially and integrating cyber and physical entities accordingly.

Despite of differences in evolution strategies, both of the iteration path and convergence path indicate that the implementation of CPS in shop-floor level is a dynamic process. Moreover, in actual applications, other constraints such as management, economy, and environment should be considered during implementation. Therefore, in order to improve feasibility and increase success rate in CPS implementation, it is necessary to build a model to evaluate the maturity of CPS which helps assess implementation methods and paths.

\section{2. ACPS Maturity Model of discrete manufacturing shop floor}

As elaborated in Section 2. 1, the evolution of CPS in shop floor level may be regarded as a dynamic process with iterations in each phase. Under this circumstance, $\mathrm{Ca}$ pability Maturity Models (CMM) method can be introduced to conceptualize and measure the maturity of this process.

CMM are widely applied in research and project management domain to describe the maturity of a process during development, usually in the form of specific levels. With regard to ICT domain, the most well-known CMM method is Capability Maturity Model Integration (CMMI) which accesses the maturity level of software development. CMMI uses 5 levels to access the maturity of development stages from initial, managed, defined and quantitatively managed to optimizing [13-15]. Recently, several researches have addressed maturity models for the assessment of enterprises in smart manufacturing domain. A maturity model comprises 9 dimensions and 62 items was proposed to assess Industry 4.0 maturity [16]. China Electronics Standardization Institute also presented a 2-dimension model with 27 criteria to access smart manufacturing capability maturity in enterprises [17]. Similarly, as CMMI, these maturity models use quantitative numbers from 1-5 to access the maturity level of each criterion. Despite simplified calculation process of this approach, the relative importance weights of different criteria are not considered. Moreover, since the quantitative judgments mostly depend on the subjective opinion of assessors, accurate results usually cannot be acquired in the context of uncertainty and vagueness in real practice.

With the purpose of establishing maturity models of CPS implementation process in discrete manufacturing shop floor, the following principles should be addressed in concerning aspects and selecting indicators for the framework:

1. Relevance. The aspects and indicators chosen in the maturity framework must be closely related to the characteristics of CPS in shop-floor level. Irrelevant indicators need to be eliminated beforehand.

2. Operability. The numbers of aspects and indicators should be limited to lower complexity for further deduction and computation procedure. As far as qualitative indicators are concerned, it should be feasible to determine relative importance by pairwise comparisons using AHP.

3. Scalability. The aspects and indicators could be 
expanded and modified in accordance with different application domains or scenarios, meanwhile, improved by the implementation experiences and advancement of technology.
Based on the principles above, the CPS Maturity Model (CPSMM) is presented in Table lincluding aspects, indicators and their explanations.

CPS Maturity Model (CPSMM)

\begin{tabular}{|c|c|c|}
\hline Aspects & Indicators & Explanation \\
\hline \multirow{3}{*}{$\begin{array}{l}\text { Sensibil- } \\
\text { ity }\end{array}$} & Process Sensibility (PS) & ability to sense process related data in real time \\
\hline & $\begin{array}{l}\text { Equipment-State Sensibility } \\
(\mathrm{SS})\end{array}$ & ability to acquire the state of equipment timely \\
\hline & Environment Sensibility (ES) & to be aware of the environment during production \\
\hline \multirow{4}{*}{$\begin{array}{l}\text { Interac- } \\
\text { tivity }\end{array}$} & Field Networkability (FN) & the networkability of shop floor field \\
\hline & $\begin{array}{l}\text { Machine - Machine Communi- } \\
\text { cation(MMC) }\end{array}$ & communication among working machines \\
\hline & $\begin{array}{l}\text { Human -Machine Collaboration } \\
\text { (HMC) }\end{array}$ & collaboration between human and machines \\
\hline & $\begin{array}{l}\text { System - System Integration } \\
\text { (SSI) }\end{array}$ & integration of related information systems, e. g. MES\& PDM \\
\hline \multirow{4}{*}{$\begin{array}{l}\text { Automa- } \\
\text { tion }\end{array}$} & Machinery Automation (MA) & deployment of automated machines/systems in shop-floors \\
\hline & Logistics Automation (LA) & $\begin{array}{l}\text { ability of automatic materials/components/ products handling and trans- } \\
\text { portation in shop-floors }\end{array}$ \\
\hline & $\begin{array}{l}\text { Process Planning and Schedul- } \\
\text { ing (PPS) }\end{array}$ & Planning and Scheduling of production process \\
\hline & Coordination Control (CC) & ability to control physical production components cooperatively \\
\hline \multirow{4}{*}{$\begin{array}{l}\text { Flexibil- } \\
\text { ity }\end{array}$} & Cell Modularity (CM) & modularity and composability of working cells \\
\hline & $\begin{array}{l}\text { Component Re-configurabil- } \\
\text { ity }(\mathrm{CR})\end{array}$ & interchangeability of equipment and components \\
\hline & Tooling Flexibility (TF) & flexibility of tools and clamping \\
\hline & Process Adaptability (PA) & $\begin{array}{c}\text { competence of adaptation responding to changes with regard to orders, } \\
\text { materials, equipment variation, etc. }\end{array}$ \\
\hline \multirow[t]{4}{*}{$\begin{array}{l}\text { Reliabil- } \\
\text { ity }\end{array}$} & Equipment Maintenance (EM) & $\begin{array}{l}\text { ensuring the equipment reliability by monitoring, detecting and main- } \\
\text { taining methods. }\end{array}$ \\
\hline & Malfunction Diagnosis (MD) & diagnosis of malfunctions in production process \\
\hline & Quality Assurance (QA) & on-line products quality inspection and controlling \\
\hline & Cyber Security(CS) & the security level of information systems \\
\hline
\end{tabular}

\section{Evaluation Method and Procedure of CPSMM}

To apply the CPSMM model, the following must be addressed:

1) The weight of indicators. Different aspects and indicators may represent uneven importance weights regarding the total maturity of evaluating objects. To assess the importance weights among different aspects and indicators, a group-decision Analytic Hierarchy Process (AHP) method is proposed which will be elaborated in Section 3. 1 .
2) The value of indicators. Traditional maturity models which mentioned above usually assign the values of indicators by 5 stages with numerical numbers from 1 to 5 . Considering the uncertainty and vagueness of practical applications, a more appropriate way of representing values of indicators with Linguistic Variables which can further transform into Triangular Fuzzy Numbers (TFNs) is presented. The details are presented in Section 3. 2.

The general procedure of proposed approach is illustrated in Fig. 2.

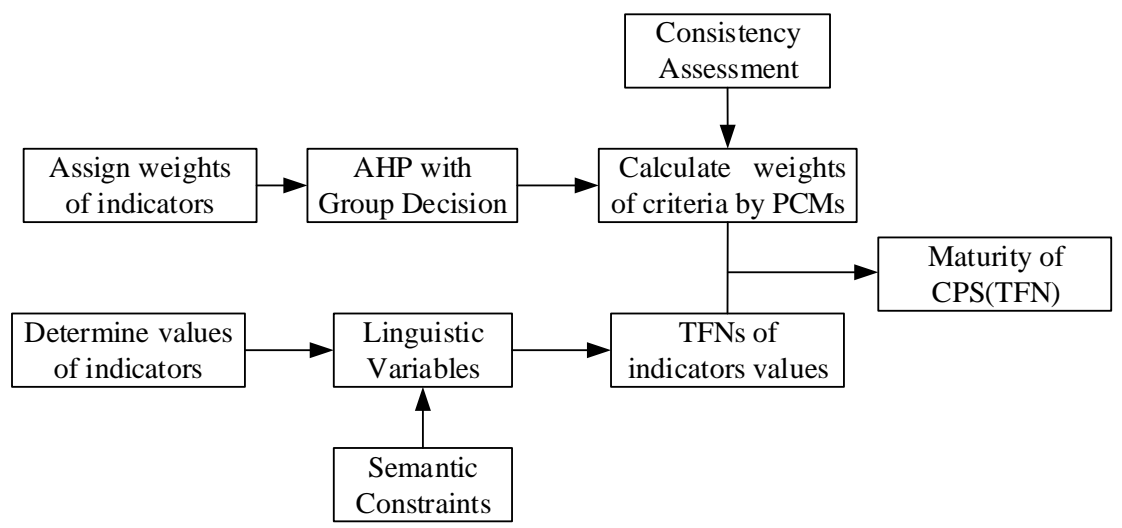

Fig. 2 General Evaluation Method and Procedure of CPSMM 


\section{1. Assigning the weights of indicators by group AHP}

The decision-making on CPS implementation in shop floor level should be considered as Multiple Criteria Decision-Making (MCDM) problem. AHP is a feasible method with regard to MCDM researches in manufacturing domain, such as AR devices application evaluation [18], supplier selection in automotive industry [19], etc. However, questions still remain in using AHP while deal with qualitative criterions as single specialist's judgment could be inaccurate or biased. Moreover, trade-offs are often faced between algorithm complexity and operation feasibility.

This paper proposed an improved AHP based architecture of assigning weights on shop floor CPS maturity model (CPSMM), in which group-decision method is introduced and detailed algorithm process is presented in the cause of improving feasibility as well as lowing algorithm complexity.

In order to determine the weights of CPSMM elements, the proposed CPSMM can be transformed to an AHP model with 2-level criteria, where the aspects of CPSMM be regarded as the key criteria level, the indicators be regarded as the secondary criteria level. That is to say, the key criteria contain 6 elements, namely, Sensibility, Interactivity, Automation, Flexibility, and Reliability. The secondary criteria are the sub-criteria of the key criteria. Take key criterion Sensibility for example, its sub-criteria are Process Sensibility Equipment-State Sensibility, Environment Sensibility. The AHP model of the CPSMM is illustrated in Fig. 3.

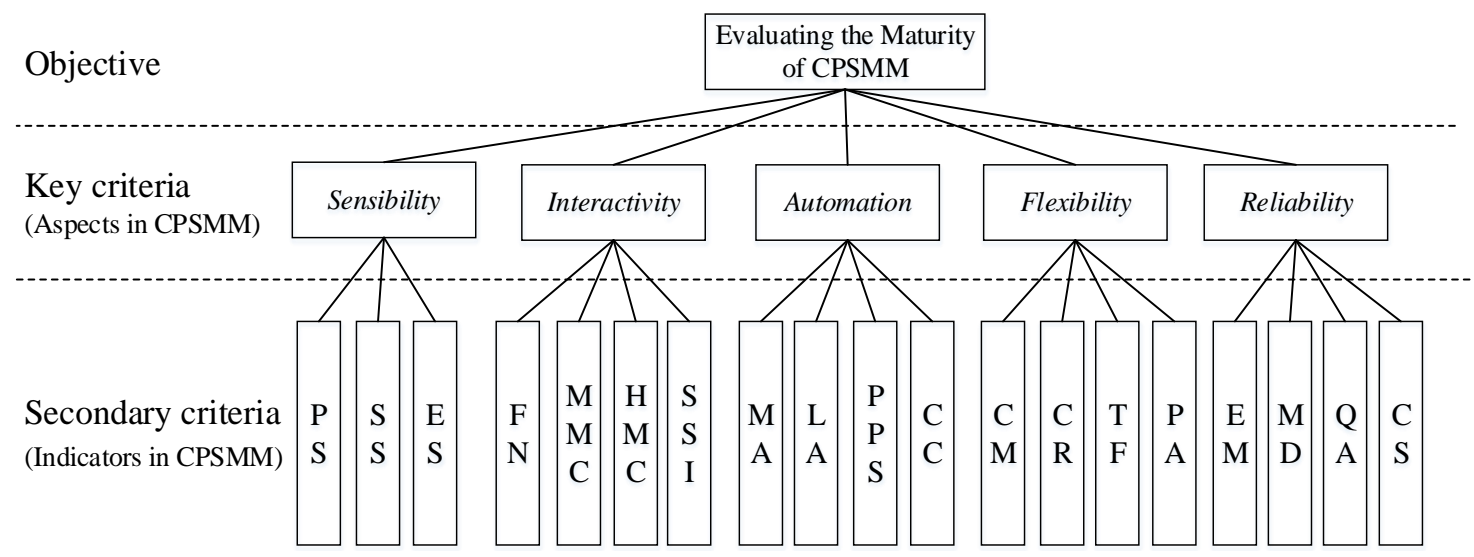

Fig. 3 The framework of CPS evaluation based on AHP(CPS-AHP)

The steps of assigning criteria weights based on group AHP are as follows:

STEP 1: Establish decision group. Build the decision group $G(m)$ with different related specialties. $m$ is the number of experts. As far as CPS is concerned, group $G(m)$ should comprise experts range from Manufacturing Technology, Information and Communication Technology, to Enterprise Management.

STEP 2: Construct Pairwise Compare Matrixes (PCMs). PCMs are constructed by 1-9 scale proposed by Satty [20].

$$
C k=\left[\begin{array}{ccc}
C_{11}^{k} & \ldots & C_{1 n}^{k} \\
\ldots & C_{i j}^{k} & \ldots \\
C_{n 1}^{k} & \ldots & C_{n n}^{k}
\end{array}\right],
$$

where: $C_{i j}^{k}$ is the importance scale of criterion compared to criterion $j$ judged by expert $k$ from $G ; n$ is number of criteria need to be compared.

STEP 3: Synthesize group PCMs decision. Assuming the experts from group $\mathrm{G}$ have equal decision priority, based on the Geometric Mean Method proposed in [21], the synthetic importance scale of group $G$ can be calculated by:

$$
C_{i j}^{G}=\left(C_{i j}^{1} C_{i j}^{2} \ldots C_{i j}^{m}\right)^{1 / m},
$$

The corresponding PCMs of $G$ is $C^{G}$ :

$$
C^{G}=\left[\begin{array}{ccc}
C_{11}^{G} & \ldots & C_{1 n}^{G} \\
\ldots & C_{i j}^{G} & \ldots \\
C_{n 1}^{G} & \ldots & C_{n n}^{G}
\end{array}\right] .
$$

STEP 4: Calculate PCMs of $C^{G}$.

1. Normalize $C^{G}$ by columns to get $C^{\mathrm{N} G}$,

$$
\begin{aligned}
C^{\mathrm{N} G} & =\left[\begin{array}{ccc}
C_{11}^{\mathrm{N} G} & \ldots & C_{1 n}^{\mathrm{N} G} \\
\ldots & C_{i j}^{\mathrm{N} G} & \ldots \\
C_{n 1}^{\mathrm{N} G} & \ldots & C_{n n}^{\mathrm{N} G}
\end{array}\right], \text { where: } \\
C_{i j}^{\mathrm{N} G} & =\frac{C_{i j}^{G}}{\sum_{i=1}^{n} C_{i j}^{G}} .
\end{aligned}
$$

2. Calculate the eigenvector $W$ of $C^{G}$, let $W=\left(w_{1}, \ldots w_{i}, \ldots w_{n}\right)^{T}$, then, $w_{i}=\frac{\sum_{j=1}^{n} C_{i j}^{N G}}{n}, \sum_{i=1}^{n} w_{i}=1$

3. Calculate the eigenvalue $\lambda_{\max }$ of $C^{G}$ :

$$
\lambda_{\max }=\sum_{i=1}^{n} \frac{\left(C^{G} W\right)_{i}}{n W_{i}}
$$




\section{STEP 5: Consistency Test.}

Define $C I$ as a consistency index of $C^{G}$, where:

$$
C I=\frac{\lambda_{\max }-n}{n-1}
$$

To test the consistency of PCM $C^{G}$, using formula:

$$
C R=\frac{C I}{R I},
$$

where: $R I$ is Radom Index [20]. If $C R<0.1$, then $C^{G}$ is considered as consistent, $W$ can be regarded as weight vector of criteria. Otherwise, the PCMs need be adjusted.

STEP 6: Repeat STEP 1 -STEP 5 to calculate the weight vector of criteria in next level. The final weights of indicator in CPSMM $w_{i}$ can be calculated by $w_{i}=w_{k} \bullet w_{s}$, where $w_{k}$ is importance weight of key criterion $k, w_{s}$ is the importance weight of secondary criterions.

\section{2. TFNs of Linguistic Variables Set with Semantic Grad- ing Constraints}

In the proposed CPSMM model, linguistic variables which ease the evaluation of criteria are introduced to replace numerical numbers. The Linguistic Variables Set is defined as following:

$$
\begin{aligned}
& L(v)= \\
& =\{\text { very low, low, medium, high, very high }\} .
\end{aligned}
$$

Triangular fuzzy functions are then introduced to transform Linguistic Variables to Triangular Fuzzy Numbers (TFNs).

Consider $M=(l, m, u)$ on $R$ to be a TFN, its membership function $\mu_{M}(x): R \rightarrow[0,1]$ is:

$$
\mu_{M}(x)= \begin{cases}\frac{x-l}{m-l}, & l \leq x \leq m \\ \frac{u-x}{u-m}, & m \leq x \leq u \\ 0, & \text { otherwise }\end{cases}
$$

With regard to CPSMM model, the membership functions of Linguistic Variables Set are defined in Fig. 4.

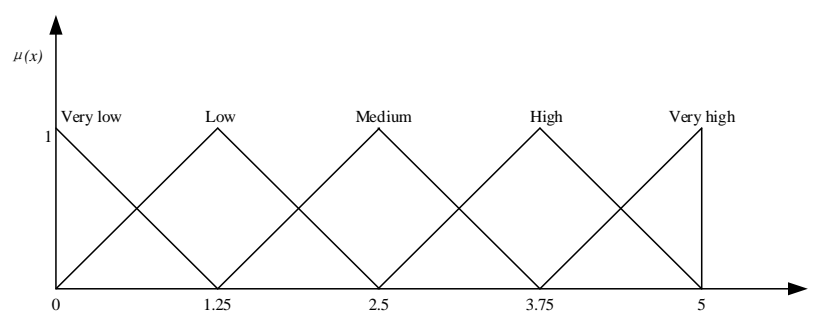

Fig. 1 Membership Functions of TFNs of Linguistic Variables Set

As depicted in Fig. 4, the evaluation value of criteria which represented by Linguistic Variables can be further transform into TFNs. However, difficulties still remained when assessors try to determine which Linguistic Variable should be assigned to specific evaluation objects. To help and also constrain the objective judgments of assessors, semantic grading constraints are proposed in case of determining the Linguistic Variables.

Take Machinery Automation (MA) criterion for example, as we know, its maturity levels are correlated with key features knowledge which could be synthesized by cognition on implementation experiences and practices. Hence,

\begin{tabular}{|c|c|c|}
\hline Semantic Constraints & $L(v)$ & TFN \\
\hline Almost all manufacturing processes are automatic & Very high & $(3.75,5,5)$ \\
\hline $\begin{array}{l}\text { Large scale of manufacturing processes are applied automatic ma- } \\
\text { chinery }\end{array}$ & High & $(2.5,3.75,5)$ \\
\hline Key processes are deployed with machine tools and robotics, etc & Medium & $(1.25,2.5,3.75)$ \\
\hline Parts of work stations are half-automatic & Low & $(0,1.25,2.5)$ \\
\hline Most of manufacturing processes are handled manually & Very low & $(0,0,1.25)$ \\
\hline
\end{tabular}
these knowledge can be transformed to semantic constraints which help to determine Linguistic Variables $L(v)$. Then, $L(v)$ can be represented by TFNs. As illustrated in Table 2.

The Semantic Grading Constraints of Machinery Automation (MA)

Using the same method, the semantic constraints of other criteria can also be established which further mapped to TFNs. By synthesizing the weight of and the value (TFNs) of criteria, the final maturity of CPSMM is achieved.

$$
M_{C P S M M}=\sum_{i=1}^{n} w_{i} \cdot v_{i},
$$

where: $M_{C P S M}=$ the maturity of CPSMM represented by TFN; $w_{i}=$ the importance weight of criterion; $v_{i}=$ the value of criterion represented by TFN. The $M_{C P S M M}$ calculated from the formula (10) is still a triangular fuzzy number (TFN) which cannot measure and evaluate the final maturity clearly. So the comparison between the $M_{C P S M M}$ and the TFNs of the semantic variable $L(v)$ are needed to translate the comparison result into the final evaluation semantics.

According to the comparison rules of the TFNs proposed by literature [22], compare the two fuzzy number $A_{1}=\left(l_{1}, m_{1}, u_{1}\right)$ and $A_{2}=\left(l_{2}, m_{2}, u_{2}\right)$, and the followings are defined:

$$
V\left(A_{1} \geq A_{2}\right)=1, \text { iff } m_{1} \geq m_{2},
$$




$$
V\left(A_{2} \geq A_{1}\right)=\operatorname{hgt}\left(A_{1} \cap A_{2}\right)=\frac{\left(l_{1}-u_{2}\right)}{\left(m_{2}-u_{2}\right)}-\left(m_{1}-l_{1}\right)
$$

where: $V \in\left(\begin{array}{ll}0 & 1\end{array}\right)$, which represents the comparison credibility of the TFNs and can be converted to a percentage.

Then the final evaluation semantics can be described as:

The CPSMM maturity $L(v)$ of this workshop is $[v]$.

\section{Illustrative example}

From 2015, China has started its "China Manufacturing 2025" strategy to promote Smart Manufacturing paradigm. Since then, some leading manufacturers have engaged in CPS researches and implementations especially in shop floor level. In this research, the proposed approach is applied in a gear manufacturing shop floor located in a coastal province of China to demonstrate the calculating procedure of CPSMM. In order to reduce labor and improve quality and efficiency, the workshop made intelligent update of the existing production conditions, mainly including: a new intelligent $\mathrm{CNC}$ machining machine, multi-industrial robot assembly workstations, an AGV car for the logistics transportation in the workshop, the industrial wired network to realize the interconnection of the processing equipment, the wireless sensor network based on ZigBee to monitor the environment such as the temperature, the noise in the workshop as well as the application of MES, APS, SCADA system for workshop process management and control, and online equipment testing for quality control.

The traditional qualitative evaluation showed that the company's workshop after updating was of a higher de- gree of smart maturity, but failed to evaluate the specific refined indicators. Based on the proposed method in this paper, the shop maturity is revaluated, whose process is as follows.

1. Calculate the CPSMM criteria weights.

To demonstrate the group-AHP method of calculating key criteria weights, experts group $\mathrm{G}$ is established containing 3 specialists from Manufacturing Technology, Information and Communication Technology, to Enterprise Management. The Pairwise Compare Matrixes (PCMs) are constructed by experts respectively using 1-9 scale compare method [20].

By STEP 1-STEP3 in Section 3.1, the group PCM $C^{G}$ is synthesized as follows:

$$
C^{G}=\left[\begin{array}{lllll}
1.00 & 1.44 & 0.79 & 1.06 & 0.79 \\
0.69 & 1.00 & 0.94 & 1.44 & 1.26 \\
1.26 & 1.06 & 1.00 & 1.59 & 1.26 \\
0.94 & 0.69 & 0.63 & 1.00 & 1.06 \\
1.26 & 0.79 & 0.79 & 0.94 & 1.00
\end{array}\right] .
$$

According to STEP 4-STEP 5, eigenvector $W$, eigenvalue $\lambda_{\max }$ and $C R$ are calculated as:

$$
\begin{aligned}
& W=(0.200,0.207,0.239,0.167,0.187)^{T}, \\
& \lambda_{\text {max }}=5.071, \\
& C R=0.016<0.1 .
\end{aligned}
$$

Because $C R=0.016<0.1$, then $W$ can be regarded as importance weights vector of criteria, as illustrated in Table 3 .

Table 3

Importance weights of key criteria

\begin{tabular}{|c|c|c|c|c|c|}
\hline Criteria & Sensibility & Interactivity & Automation & Flexibility & Reliability \\
\hline Weight & 0.200 & 0.207 & 0.239 & 0.167 & 0.187 \\
\hline
\end{tabular}

Using the same procedure, the importance weights of secondary criteria of is calculated. The final weights of indicators of CPSMM are then calculated by STEP 6 in Section 3. 1.

2. Measure indicators with TFNs

As proposed in Section 3.2, the value of indicators is measured by linguistic variables during assessment process through questionnaires and field studies. The linguistic variables are then transformed to TFNs with the guide of semantic constraints. Indicators' values of target shop floor are given in Tab 4, where $v_{i}$ is the value of criterion $i$ represented by TFN, $w_{i}$ is the importance weight of criterion $i$.

Table 4

Values and global weights of CPSMM indicators

\begin{tabular}{|c|c|c|c|c|}
\hline Indicators of CPSMM & \multicolumn{2}{|c|}{$v_{i}$} & \multirow{2}{*}{$w_{i}$} \\
\cline { 2 - 5 } & $l$ & $m$ & \multicolumn{2}{c|}{$u$} \\
\hline Process Sensibility (PS) & 1.25 & 2.5 & 3.75 & 0.084 \\
\hline Equipment-State Sensibility (SS) & 2.5 & 3.75 & 5 & 0.070 \\
\hline Environment Sensibility (ES) & 3.75 & 5 & 5 & 0.046 \\
\hline Field Networkability (FN) & 2.5 & 3.75 & 5 & 0.068 \\
\hline Machine - Machine Communication(MMC) & 1.25 & 2.5 & 3.75 & 0.035 \\
\hline Human -Machine Collaboration (HMC) & 0 & 1.25 & 2.5 & 0.043 \\
\hline System - System Integration (SSI) & 1.25 & 2.5 & 3.75 & 0.060 \\
\hline Machinery Automation (MA) & 3.75 & 5 & 5 & 0.067 \\
\hline
\end{tabular}




\begin{tabular}{|c|c|c|c|c|}
\hline Indicators of CPSMM & \multicolumn{2}{|c|}{$v_{i}$} & \multirow{2}{*}{$w_{i}$} \\
\cline { 2 - 4 } & $l$ & $m$ & $u$ & \multirow{2}{*}{0.050} \\
\hline Logistics Automation (LA) & 2.5 & 3.75 & 5 & 0.062 \\
\hline Process Planning and Scheduling (PPS) & 2.5 & 3.75 & 5 & 0.060 \\
\hline Coordination Control (CC) & 0 & 1.25 & 2.5 & 0.028 \\
\hline Cell Modularity (CM) & 1.25 & 2.5 & 3.75 & 0.038 \\
\hline Component Re-configurability(CR) & 1.25 & 2.5 & 3.75 & 0.048 \\
\hline Tooling Flexibility (TF) & 0 & 1.25 & 2.5 & 0.052 \\
\hline Process Adaptability (PA) & 1.25 & 2.5 & 3.75 & 0.054 \\
\hline Equipment Maintenance (EM) & 3.75 & 5 & 5 & 0.041 \\
\hline Malfunction Diagnosis (MD) & 2.5 & 3.75 & 5 & 0.034 \\
\hline Quality Assurance (QA) & 3.75 & 5 & 5 & 0.050 \\
\hline Cyber Security(CS) & 1.25 & 2.5 & 3.75 & 0.062 \\
\hline
\end{tabular}

The final maturity of the target shop floor then is calculated by formula (10):

$$
M_{\text {CPSMM }}=(1.93,3.18,4.17) .
$$

As defined in Section 3.2, the triangular fuzzy number of " $L(v)=\{h i g h\} "$ is $\mathrm{TFN}=(2.5,3.75,5)$. According to the TFN comparison method proposed in Section 3.2, the credibility of $M_{C P S M M} \geq H$ is:

$$
V\left(M_{\text {CPSMM }} \geq H\right)=h g t\left(H \cap M_{\text {CPSMM }}\right)=0.746 .
$$

That is, the CPSMM maturity of this workshop is $74.6 \%$ of high level.

The maturity of the CPS in the workshop is close to the High level from the semantic maturity evaluation, due to the equipment update, automation level improvement, the enhancement of sensing/detection capabilities and some refinement indexes (Machinery Automation (MA), Environment Sensibility (ES), and Quality Assurance (QA)). While, there are still some indexes with lower level in Human-Machine Collaboration (HMC), Coordination Control (CC), and Tooling Flexibility (TF). So it is suggested for the workshop that the AR/MR technology be applied to improve the interactive capabilities of Human and Machine, the intelligent algorithms such as reinforcement learning be introduced to improve the coordination control capability and the modular tooling be used to improve the tooling flexibility.

\section{Conclusions}

Assessing the CPS maturity in discrete shop floor gives enterprises a feasible approach to be aware of the implementation status thereby to improve its weak aspects. This paper presents a maturity model of CPS in discrete shop floor level named CPSMM which contains 5 aspects and 19 indicators. Different from the existing maturity models, the CPSMM model introduced group AHP method to assign the importance weights of indicators, and then the linguistic variable and TFNs are defined and synthesized to represent the value of indicators quantitatively with the guide of semantic constraints. At last, the above model is applied in a gear manufacturing shop floor located in a coastal province of China to demonstrate the procedure of the given approach.

The further research will focus on the synthesis of different methods combine with the proposed maturity model to achieve better result of assessment. In addition, An Extensible architecture for the decision support system based on the given model needs to be construct to simulate and compare different implementation plan during CPS introduction.

\section{Acknowledgements}

This work was financially supported by the National Defense Science and Technology Project Foundation (No.0106142), and MOE (Ministry of Education in China) Project of Humanities and Social Sciences (No.17YJC630139). The supports are gratefully acknowledged.

\section{Statement}

The author(s) declare(s) that there is no conflict of interests and the mentioned received funding in the Acknowledgement section did not lead to any conflicts of interest regarding the publication of this manuscript.

\section{References}

1. Lu, Y.; Morris, K. C.; Frechette, S. 2016. Current Standards Landscape for Smart Manufacturing Systems $1-39$. http://dx.doi.org/10.6028/NIST.IR.8107.

2. Weichhart, G. et al. 2016. Challenges and current developments for Sensing, Smart and Sustainable Enterprise Systems. Computers in Industry 79: 34-46. https://doi.org/10.1016/j.compind.2015.07.002.

3. Shariatzadeh, N. et al. 2016. Integration of Digital Factory with Smart Factory Based on Internet of Things, Procedia CIRP, 50: 512-517. https://doi.org/10.1016/j.procir.2016.05.050.

4. Wang, S. et al. 2016. Towards smart factory for industry 4.0: a self-organized multi-agent system with big data based feedback and coordination, Computer Networks 101: $158-168$ https://doi.org/10.1016/j.comnet.2015.12.017.

5. Sunder, S. S. 2012. Foundations for innovation in cyber-physical systems $[\mathrm{C}] / /$ proceedings of the NIST 
CPS Workshop, Chicago, IL, USA. 13. https://doi.org/10.1007/s13398-014-0173-7.2.

6. Monostori, L. 2014. Cyber-physical Production Systems: Roots, Expectations and R\&D Challenges, Procedia CIRP, 17: 9-13. https://doi.org/10.1016/j.procir.2014.03.115.

7. Babiceanu, R. F.; Seker, R. 2016. Big Data and virtualization for manufacturing cyber-physical systems: A survey of the current status and future outlook, Computers in Industry, 81: 128-137. https://doi.org/10.1016/j.compind.2016.02.004.

8. Lee, J.; Bagheri, B.; Kao, H. 2015. A Cyber-Physical Systems architecture for Industry 4.0-based manufacturing systems, Manufacturing Letters, 3: 18-23. https://doi.org/10.1016/j.mfglet.2014.12.001.

9. Michniewicz, J.; Reinhart, G. 2016. Cyber-PhysicalRobotics - Modelling of modular robot cells for automated planning and execution of assembly tasks, Mechatronics, 34: 170-180. https://doi.org/10.1016/j.mechatronics.2015.04.012.

10. Pirvu, B.; Zamfirescu, C.; Gorecky, D. 2016. Engineering insights from an anthropocentric cyber-physical system: A case study for an assembly station, Mechatronics, 34: 147-159. https://doi.org/10.1016/j.mechatronics.2015.08.010.

11. Dworschak, B.; Zaiser, H. 2014. Competences for Cyber-Physical Systems in Manufacturing - First Findings and Scenarios, Procedia Cirp 25: 345-350. https://doi.org/10.1016/j.procir.2014.10.048.

12. Liu, C.; Jiang, P. 2016. A Cyber-Physical System Architecture in Shop Floor for Intelligent Manufacturing, Procedia CIRP 56: 372-377. https://doi.org/10.1016/j.procir.2016.10.059.

13. Pane, E. S.; Sarno, R. 2015. Capability Maturity Model Integration (CMMI) for Optimizing Object-Oriented Analysis and Design (OOAD), Procedia Computer Science 72: 40-48. https://doi.org/10.1016/j.procs.2015.12.103.

14. Gudergan, G. et al. 2015. Evaluating the Readiness to Transform Towards a Product-service System Provider by a Capability Maturity Modelling Approach, Procedia CIRP 30: 384-389. https://doi.org/10.1016/j.procir.2015.02.134.

15. Torrecilla-Salinas, C. J. et al. 2016. Agile, Web Engineering and Capability Maturity Model Integration: A systematic literature review, Information and Software Technology 71: 92-107. https://doi.org/10.1016/j.infsof.2015.11.002.

16. Schumacher, A.; Erol, S.; Sihn, W. 2016. A Maturity Model for Assessing Industry 4.0 Readiness and Maturity of Manufacturing Enterprises, Procedia CIRP 52: 161-166. https://doi.org/10.1016/j.procir.2016.07.040.

17. China Academy of electronic technology standardization, Intelligent manufacturing capability maturity model white paper. 2017.

18. Elia, V.; Gnoni, M. G.; Lanzilotto, A. 2016. Evaluating the application of augmented reality devices in manufacturing from a process point of view: An AHP based model, Expert Systems with Applications 63: 187-197. https://doi.org/10.1016/j.eswa.2016.07.006.

19. Dweiri, F. et al. 2016. Designing an integrated AHP based decision support system for supplier selection in automotive industry, Expert Systems with Applications, 62: 273-283. https://doi.org/10.1016/j.eswa.2016.06.030.

20. Saaty, T. L. 1990. How to Make a Decision: The Analytic Hierarchy Process, European Journal of Operational Research 48(1): 9-26. https://doi.org/10.1016/0377-2217(90)90057-I.

21. Akaa, O. U. et al. 2016. A group-AHP decision analysis for the selection of applied fire protection to steel structures, Fire Safety Journal 86: 95-105. https://doi.org/10.1016/j.firesaf.2016.10.005.

22. Agrawal, S. et al. 2017. An extended Fuzzy-AHP approach to rank the influences of socialization -externalization-combination-internalization modes on the development phase, Applied Soft Computing 52(C): 505518.

http://dx.doi.org/10.1016/j.asoc.2016.10.017.

Qingmeng Tan, Yifei Tong, Shaofeng Wu, Dongbo Li

EVALUATING THE MATURITY OF CPS IN DISCRETE MANUFACTURING SHOP-FLOOR: A GROUP AHP METHOD WITH FUZZY GRADE APPROACH

S u m m a r y

Discrete manufacturing faces challenges to improve quality and efficiency, reduce labour and costs, as well as the shifting market from customized manufacturing to short-series production, just to name a few. CPS which combines physical world and cyber world is a key technology of coping these challenges particularly in the shop floor level. However, due to its complexities and heterogeneities, CPS development and implementation in discrete manufacturing field is still in rudimentary phase which requires feasible methods to evaluating the maturity of current system. This paper proposes an evolution process of CPS in discrete manufacturing which can be characterized by two-dimensional perspectives concerning MT (manufacturing technology) and ICT (information and communication technology) elements. A maturity model (CPSMM) is presented to conceptualize the maturity of CPS implementation process in discrete manufacturing shop floor. The importance weights of indicators in the maturity model are acquired based on AHP with group decision. A semantic grading approach is proposed to guide and constrain accessing process which allows using triangle fuzzy numbers. Procedures and algorithms on calculating CPS-AHP are given and applied in a mechanical shop floor for illustration.

Keywords: CPS, maturity, shop floor, AHP, Fuzzy.

Received August 30, 2017 Accepted February 15, 2018 